\title{
The Pennsylvania Adoption Exchange Improves Its Matching Process
}

\author{
Vincent W. Slaugh, Mustafa Akan, Onur Kesten \\ Tepper School of Business, Carnegie Mellon University, Pittsburgh, PA 15213 \\ vslaugh@cmu.edu, akan@cmu.edu, okesten@andrew.cmu.edu \\ M. Utku Ünver \\ Department of Economics, Boston College, Chestnut Hill, MA, 02467 \\ unver@bc.edu
}

\begin{abstract}
The Pennsylvania Adoption Exchange (PAE) helps case workers who represent children in state custody by recommending prospective families for adoption. We describe PAE's operational challenges using case worker surveys and analyze child outcomes through a regression analysis of data collected over multiple years. A match recommendation spreadsheet tool implemented by PAE incorporates insights from this analysis and allows PAE managers to better utilize available information. Using a discrete-event simulation of PAE, we justify the value of a statewide adoption network and demonstrate the importance of better information about family preferences for increasing the percentage of children who are successfully adopted. Finally, we detail a series of simple improvements that PAE achieved through collecting more valuable information and aligning incentives for families to provide useful preference information.
\end{abstract}

Key words: community OR; public service; matching; market design

According to the most recent report of the Children's Bureau of the US Department of Health and Human Services (2014), approximately 397,000 children in the United States are living in the foster care system, with 102,000 of them waiting for adoptive placement. In 2012, while 50,000 children were successfully adopted from foster care, approximately 23,000 were discharged due to emancipation as they reached the age of 18 without receiving a permanent home. As cataloged by Howard and Brazin (2011), numerous studies have shown that children who spend significant time in foster care or "age out" of foster care without finding a permanent family suffer from alarming levels of unemployment, homelessness, early parenthood, and incarceration. For example, Reilly (2003) reports that $41 \%$ of respondents between the ages of 18 and 25 who had aged out of the foster care system have spent time in jail. 
In response to these trends, state governments, county agencies, and non-profit organizations have devoted significant resources to providing children in foster care with permanent placements in a timely manner. Federal legislation such as the Fostering Connections to Success and Increasing Adoptions Act of 2008 has mandated and reinforced these efforts. The state of Pennsylvania funds the Pennsylvania Adoption Exchange (PAE), which was established in 1979 to support county and nonprofit agencies as they attempt to find adoptive families for children who are difficult to place due to attributes such as age or special needs. In addition to listing children on a website and hosting in-person matching events, PAE maintains detailed data on children and the preferences of families. PAE is mandated by the state to recommend matches between families and children. We collaborated with managers at the Pennsylvania Statewide Adoption and Permanency Network (SWAN), a non-profit organization responsible for administering PAE on behalf of Pennsylvania, to redesign the match recommendation process.

PAE's match recommendation function has two main goals. First, it helps overcome geographical and institutional barriers in the adoption search process, given Pennsylvania's 67 counties that are supported by 82 non-profit organizations. Second, the match recommendation system helps social workers search through extensive data on child characteristics and family preferences. Furthermore, PAE managers believe that case workers sometimes have excessively high expectations — i.e., they are waiting for the "perfect" family — so the process has an additional goal of promoting a decision-making structure.

In this paper, we investigate PAE to help increase the success of match attempts. Our project contributes to an interesting and important public policy area and non-profit application of market design. We focus on simple changes that address PAE's most significant challenges, and identify key elements of the child adoption market. We have worked with PAE to collect additional information from families and children and create a spreadsheet matching tool that is now used by PAE staff to recommend families. While the idea of a computerized matching tool for ranking families is not new, we believe that we are the first to link its effectiveness to an increased rate of successful adoption through a discrete-event simulation of the adoption network. We are also among the first to note that the matching process may distort incentives for families in revealing their preferences truthfully and propose simple remedies.

The remainder of our paper is organized as follows. First, we provide context for the problem of a match recommendation system as it relates to research on the design of matching markets. We then characterize the challenges that PAE faces and assess the current system through case worker surveys and a regression 
analysis using child outcome data from 2005 to 2013. We describe how PAE recommends prospective families for children by comparing children's needs with family preferences on a set of approximately 100 attributes using a spreadsheet tool. Based on this understanding of PAE's role in the matching process, we analyze the value of the network and the information available to the network through a discrete-event simulation of PAE's operation. We then discuss our recommendations for the information that PAE collects, the decision rules for match recommendations, and the interaction with system participants. Finally, we conclude by summarizing the implemented improvements and possible future improvements in adoption and other similar domains.

\section{Design of Adoption Markets}

Despite the growing prominence and urgency of finding families for children in state custody, little is known about how to define, analyze, and improve the family finding process. The fundamental supply-and-demand imbalance for children of different demographic characteristics was first described using an economics framework by Landes and Posner (1978). An empirical study by Baccara et al. (2014) identifies biases in preferences of prospective adoptive families for infant adoption. They show that an adopted child's desirability to prospective families depends heavily on the child's age, gender, and race, with some of the greatest disparities accounted for by the child's race.

We view PAE as a two-sided matching market and rely on the market design literature to frame our approach to the problem while using operations research and economics techniques to improve the current recommendation practice. Early market design work focused on understanding and improving centralized clearinghouses that operate in the absence of prices and face institutional and ethical constraints. The seminal work of Gale and Shapley (1962) introduced the formal two-sided matching framework. This theory was subsequently advanced and adapted to important applications such as the design of the National Residency Matching Program in the US for matching medical school graduates to internships, residencies, and fellowships at hospitals, as described by Roth and Peranson (1999). This approach was also adapted for other applications such as the assignment of students to public schools (Abdulkadiroğlu and Sönmez 2003) and kidney exchange (Roth et al. 2005).

A recent strand of literature in matching market design has focused on introducing new ideas to improve the functioning of a centralized or decentralized matching market rather than designing new clearinghouses 
to conduct the matching. For example, Coles et al. (2010) report the introduction of a signaling tool for the academic job market for new economics PhDs. Lee et al. (2011) report an experiment to measure the effects of the use of signaling devices on online dating. Also, a new company, OrganJet, uses private jets to transport patients in order to overcome the inefficiencies of regionally isolated organ donation networks (Ata et al. 2012). A related study by Arikan et al. (2012) shows that broader sharing of the bottom 15\% of kidneys (in terms of quality) from deceased donors leads to significantly increased procurement rates for those organs.

\section{Child Adoptions in Pennsylvania}

The statewide network's primary goal is to help find permanent families for children in state custody. Children who fail to be placed upon initial attempts at the county level are provided with extra services at the state level, including match recommendations from PAE. County child welfare appropriations in Pennsylvania exceeded \$1.5 billion in fiscal year 2014-2015 (PA Department of Human Services 2015), and are largely used to support approximately 15,000 children in foster care and 2,000 children who are classified as waiting for adoption (Children's Bureau, U.S. Department of Health and Human Services 2014). Between 2007 and 2012, an average of 239 children per year registered to receive match recommendations from PAE. The Office of Children, Youth and Families (OCYF) of Pennsylvania's Department of Human Services mandates that children without an identified adoptive family must be registered with PAE within ninety days of termination of parental rights (TPR).

PAE managers believe that the best scenario for children is to be placed with a permanent family, even for a child who is about to reach the age of majority. For children who are placed, the time during which the child is a legal orphan should be minimized. Furthermore, the suitability of the family for the child is also important. In particular, some families are better prepared than others to handle children with certain needs, whether medical, behavioral, or psychological.

Prior to TPR, a county case worker seeks a suitable family for the child. If there is no clear kinship adoption possibility or potential family within the agency's local network, the CYS worker may contact SWAN and request match suggestions from PAE. The case worker must register the child with PAE in the ninety days after TPR if the worker has not identified a resource for a child. Through working with PAE coordinators, the CYS worker then receives between five and ten families to consider and pursue. After identifying interested 
families, the worker then arranges an interview and consults with a committee comprised of social workers and other professionals with diverse expertise to choose a family with which to place the child. After a series of successful visits of increasing duration and decreasing supervision, the child is placed with the family. According to Pennsylvania law, an adoption can then be finalized after six months.

To characterize the challenges facing PAE, we worked with a PAE manager to perform a survey of case workers for all active children in spring 2011 to gain a broader picture of feelings about PAE across the state. Survey recipients included both county case workers and social workers known as "child-specific recruiters" who work for private non-profit agencies and serve as an additional resource for county case workers in finding families for hard-to-place children. See the Supplemental Files for the entire survey and results.

The case workers were first asked to indicate the helpfulness of various avenues of finding families: $65 \%$ of respondents said that this never or rarely served as the initial source of prospective families for children who are successfully placed. Furthermore, no respondents strongly agreed and only $32 \%$ agreed with the statement that "PAE does a good job of recommending the most suitable families via electronic matches from the Resource Family Registry for each child." The case workers also testified to the difficulty of making placement decisions and case worker bias. More respondents agreed or strongly agreed (53\%) than disagreed or strongly disagreed $(37 \%)$ with the statement that they "know of case workers who struggle to make placement decisions for children because of emotional attachments to those children." Even more respondents agreed $(65 \%)$ than disagreed $(23 \%)$ with the statement that they "know of case workers whose personal preferences lead to negative perceptions towards some families."

Survey responses demonstrate both the ineffectiveness of the current matching system and the case workers' mistrust of match recommendations from PAE. However, case workers expressed more positive views about possible helpfulness of the registration data, with over $60 \%$ of respondents agreeing or strongly agreeing that family data is "helpful" for screening and child data is "accurate." This indicates the potential value of a statewide matching network, and motivates efforts to improve PAE's ability to help case workers find families for children in state custody.

\section{Analysis of Child Outcomes}

We reviewed registration and outcome information about children served by PAE to better understand adoption trends in Pennsylvania and the varying levels of difficulty in trying to find adoptive placements. 
PAE managers overcame significant challenges related to the decentralized nature of the adoption process in Pennsylvania to prepare this data set for our use. We are the first to analyze the relationship between child outcomes and child attributes upon registration in Pennsylvania, and the results of the analysis have provided insights about what children might require additional adoption resources and information to share with case workers as part of training on best practices.

Between 2005 and 2013, PAE assisted in the family-finding process for 1,853 children seeking adoptive families. This set of children was a subset of children in state custody with the goal of adoption; only when the matching process encounters difficulties at the county level does the search process shift to the state level. The mean age of a child upon registration with PAE was 9.41 years, and the median age was 9.63 years. Boys comprised $57.8 \%$ of all PAE registrants. Of these 1,853 children, outcomes were known for 1,514 children, as 283 were still active upon the creation of the data set in May 2013, and outcomes were missing for 56 children. Otherwise, child outcomes are known and grouped into categories, each of which has a value between 0 and 1 that is given by PAE managers. The most desirable outcome, a finalized adoption, has a value of 1 . Emancipation, which can be referred to as "aging out" of the system, is the least desirable outcome and has a value of 0 . Other positive outcomes include permanent guardianship arrangement (0.8) and living with a relative (0.7), among other scenarios. An outcome of "hold" with a foster care arrangement is considered a neutral outcome and has value of 0.5 . Other negative outcomes include placement in a residential facility (0.2) and a goal change so that the child's case worker is no longer seeking an adoptive placement for the child (0.1).

Of the children for whom outcome data are known, the county case worker succeeded in finding a finalized adoptive placement for $41.4 \%$ (627 of 1,514) of children. Another $19.1 \%$ of children have lesser positive outcomes with values of 0.7 or 0.8 . Negative outcomes with values less than or equal to 0.2 are experienced by $26.2 \%$ of children, with $12.4 \%$ of children aging out of the system. The remaining portion (13.3\%) of children have neutral outcomes. Using the values given by PAE managers, the expected outcome value for a child in the data set is 0.64 .

\section{Regression Model}

We developed a linear regression model and a logistic regression model to analyze the relationship between children's attributes when they were registered with PAE and their outcomes. Eighty-eight factors were created from registration data, and the outcome was used as the dependent variable. Specifically, the outcome 
value was used for the linear model and a binary variable with positive outcomes having value one and neutral and negative outcomes having value zero for the logit model. The square of the child's age upon registration was included to represent the increasing importance of age for older children. The date of registration was expressed in the fractional number of years after January 1, 2005. Gender was represented by a binary variable, as were whether the child had a designation of being African-American and/or Hispanic. Another binary variable represented whether the child had more than one race designation. Eighteen binary variables represented the items under the sections labeled "Educational Status" and "Special Needs" on the CY 130 forms, and an additional variable counted the number of binary variables with positive responses. The last category of variables was 61 questions in the CY 130 section labeled "Characteristics of Child," with a binary variable for each question representing a "Yes" answer. A variable that counted the number of positive responses in the section was also used.

Starting with these 88 variables, we performed a backward stepwise procedure using the Akaike information criterion for both the linear and logit models to select which variables to include in the model. Linear and logit regressions were performed on the union of the variables from these two models (Table 1). To roughly assess model performance for the linear model, rounding the predicted outcome to the nearest outcome value and classifying it as positive, negative, or neutral, the model classifies $60.0 \%$ into the correct one of three categories. Only $8.1 \%$ of children had a positive or negative outcome that was errantly predicted as the opposite outcome. All other prediction errors involved the neutral outcome. When binary child outcomes are considered for the logistic models, $75 \%$ of outcomes are correctly predicted. For children with negative/neutral outcomes, $59 \%$ were correctly predicted, and $85 \%$ were correctly predicted for children with positive outcomes.

The child's age upon registration plays a significant role in the regression model and confirms that older children become increasingly difficult to place. For the linear model, the predicted likelihood of success decreases by 0.034 per year at age eight and by 0.087 per year at age 16 . The accumulative decrease in the expected outcome compared to a newborn child is then 0.054 for an eight year-old child and 0.535 for a 16 year-old child. Although PAE managers anticipated this general trend, they found the quantification of this relationship to be very helpful as they instruct case workers around the state on best practices. Specifically, they can encourage case workers to register children with the PAE as soon as possible in the adoption process and perform a search through PAE in parallel with family reunification or other placement efforts. 
Table 1 We choose 28 factors from the available 88 factors to model child outcomes using ordinary least squares and logistic regression methods. Age upon registration, which is a negative factor for children six years of age and older, was the most important factor for predicting outcomes.

\begin{tabular}{|c|c|c|c|c|c|c|c|c|}
\hline \multirow[b]{4}{*}{ Constant } & \multicolumn{6}{|c|}{ Dependent variable: } & \multirow{4}{*}{ Freq. } & \multirow{4}{*}{ Import. } \\
\hline & \multirow{2}{*}{\multicolumn{3}{|c|}{$\begin{array}{c}\text { Outcome Value } \\
\text { Ordinary Least Squares }\end{array}$}} & \multirow{2}{*}{\multicolumn{3}{|c|}{$\begin{array}{c}\text { Outcome (Binary) } \\
\text { Logistic } \\
\end{array}$}} & & \\
\hline & & & & & & & & \\
\hline & 0.794 & $* * *$ & $(0.046)$ & 1.516 & $* * *$ & $(0.372)$ & & \\
\hline Age upon Registration (years) & 0.020 & $* *$ & $(0.009)$ & 0.102 & & $(0.075)$ & & High \\
\hline (Age upon Registration) $^{2}$ & -0.003 & $* * *$ & $(0.0005)$ & -0.017 & $* * *$ & $(0.004)$ & & High \\
\hline Registration Year (after 2005) & -0.009 & $* *$ & $(0.004)$ & -0.059 & $*$ & $(0.031)$ & & \\
\hline Male & 0.019 & & $(0.017)$ & 0.100 & & $(0.128)$ & $57.1 \%$ & High \\
\hline African-American & -0.034 & $* *$ & $(0.017)$ & -0.198 & & $(0.132)$ & $42.5 \%$ & High \\
\hline Hispanic & -0.051 & $* *$ & $(0.024)$ & -0.303 & $*$ & $(0.179)$ & $14.1 \%$ & High \\
\hline \multicolumn{9}{|l|}{ SPECIAL NEEDS } \\
\hline Mental Retardation Diagnosis & -0.109 & $* * *$ & $(0.031)$ & -0.562 & ** & $(0.230)$ & $9.0 \%$ & High \\
\hline Multiple Placement History & -0.035 & $*$ & $(0.018)$ & -0.189 & & $(0.137)$ & $45.6 \%$ & Medium \\
\hline Drug Exposed Infant & -0.020 & & $(0.026)$ & -0.100 & & $(0.202)$ & $11.6 \%$ & Medium \\
\hline Emotional Disability & -0.019 & & $(0.022)$ & -0.071 & & $(0.162)$ & $20.2 \%$ & Medium \\
\hline General Education & 0.064 & $* * *$ & $(0.019)$ & 0.353 & ** & $(0.146)$ & $37.1 \%$ & \\
\hline Siblings & 0.085 & $* * *$ & $(0.019)$ & 0.465 & $* * *$ & $(0.143)$ & $47.3 \%$ & High \\
\hline \multicolumn{9}{|l|}{ CHILD CHARACTERISTICS } \\
\hline Blind & -0.164 & * & $(0.085)$ & -0.899 & & $(0.611)$ & $1.0 \%$ & Medium \\
\hline Uses Foul or Bad Language & -0.118 & $* * *$ & $(0.027)$ & -0.613 & $* * *$ & $(0.194)$ & $15.0 \%$ & Medium \\
\hline History of Running Away & -0.086 & ** & $(0.043)$ & -0.443 & & $(0.321)$ & $4.2 \%$ & High \\
\hline Desires Contact with Siblings & -0.079 & $* * *$ & $(0.020)$ & -0.443 & $* * *$ & $(0.152)$ & $59.4 \%$ & Low \\
\hline In Contact with Former Foster Family & -0.064 & $* * *$ & $(0.022)$ & -0.353 & ** & $(0.162)$ & $18.8 \%$ & Low \\
\hline Rejects Father Figures & -0.061 & $* *$ & $(0.031)$ & -0.345 & & $(0.230)$ & $8.5 \%$ & Low \\
\hline Difficulty Accepting and Obeying Rules & -0.061 & $* * *$ & $(0.022)$ & -0.337 & $* *$ & $(0.160)$ & $36.9 \%$ & Low \\
\hline In Contact with Birth Parents & -0.058 & $* * *$ & $(0.020)$ & -0.327 & $* *$ & $(0.153)$ & $26.0 \%$ & Low \\
\hline Num. of Characteristics Present & 0.007 & $* * *$ & $(0.003)$ & 0.034 & $*$ & $(0.020)$ & & \\
\hline Parent(s) with Criminal Record & 0.017 & & $(0.018)$ & 0.087 & & $(0.138)$ & $51.6 \%$ & Low \\
\hline Difficulty Relating to Others & 0.018 & & $(0.022)$ & 0.101 & & $(0.168)$ & $31.0 \%$ & Low \\
\hline Speech Problems & 0.024 & & $(0.024)$ & 0.176 & & $(0.191)$ & $18.4 \%$ & Low \\
\hline Previous Adoption or Disruption & 0.038 & * & $(0.021)$ & 0.220 & & $(0.155)$ & $24.1 \%$ & Low \\
\hline Strong Ties to Foster Family & 0.041 & $* *$ & $(0.018)$ & 0.226 & * & $(0.134)$ & $54.2 \%$ & Low \\
\hline Vision Problems & 0.042 & * & $(0.023)$ & 0.224 & & $(0.175)$ & $17.1 \%$ & Low \\
\hline High Achiever & 0.054 & $* *$ & $(0.025)$ & 0.283 & & $(0.190)$ & $13.1 \%$ & Low \\
\hline Observations & & 1,5 & 514 & & 1,514 & & & \\
\hline $\mathrm{R}^{2}$ & & 0.3 & 345 & & & & & \\
\hline Adjusted $\mathrm{R}^{2}$ & & & 333 & & & & & \\
\hline Akaike Inf. Crit. & & & 1,697 & & & & & \\
\hline
\end{tabular}

Note: The values in parentheses indicate the standard deviation. Italicized variable names refer to "Characteristics of Child" questions on CY 130 form. Also, ${ }^{*} \mathrm{p}<0.1 ;{ }^{* *} \mathrm{p}<0.05 ;{ }^{* * *} \mathrm{p}<0.01$. "Freq." refers to an attribute's prevalence among the observation. "Import." refers to the existing default weight given to the attribute before our analysis.

Mental retardation, which had a coefficient of -0.109 for the linear model, was the most negative of the significant special needs factors from the "Child's Statuses" section of the CY 130 form for both models. Two special needs factors — having siblings and attending school in a general education setting — had 
statistically significant positive coefficients for both models. For the "Characteristics of Child" attributes, the result that surprised PAE managers was a linear regression coefficient of -0.118 for children who use foul or bad language, which was also significantly negative for the logit model. PAE managers found this information valuable to share with case workers as part of training on how to identify challenges to a successful placement. Other factors that were significantly negative with $95 \%$ confidence for both models were a difficulty accepting and obeying rules, a desired contact with siblings, contact with birth parents, and contact with the former foster family. The child's gender was revealed not to have a significant effect in either model, but outcome value decreases of 0.034 and 0.051 (for the linear model) were expected for children with African-American and Hispanic designations, respectively, although the logit model did not determine designation as African-American to have a significant effect.

To understand how the regression results compare to managerial intuition about the different factors' relative importance, we compare them to PAE managers' existing classification the factors. As part of a previous attempt to create a family ranking tool that had encountered difficulties, managers divided the factors from the CY 130 form based on their perceived importance into groups with 15 factors as "high," 18 factors as "medium," and 41 factors as "low." Of the ten significant factors with the most negative OLS coefficient, managers had assessed three of the factors (Hispanic, mental retardation diagnosis, and history of running away) as of high importance and two factors (blind and uses foul or bad language) as of medium importance. They classified the remaining five factors as of low importance. These five factors, which include two behavioral traits and three related to a child's social connections, merit closer attention and a higher weight in the matching process to help identify families more suited to a child's needs. As a result of this analysis, they ultimately decided to reclassify almost all significant $(p<0.1)$ factors with a negative OLS coefficient as of high importance in the match assessment tool, which we will further discuss later in the paper. The lone exception to this rule was multiple placement history, which remained at medium priority due to the low magnitude of its coefficient. PAE managers particularly appreciated the suggestion to increase the importance of a factors related to a child's social connections, as they identified those connections as a frequent obstacles to adoption.

PAE managers identified four other characteristics, which represent some of the most severe behavioral needs on the CY 130 form (e.g., abusing animals), as of high importance that did not appear as significant factors. However, increased managerial attention to finding families related to these children's needs might 
Table 2 PAE managers use data on 76 attributes to recommend families for children. Weights displayed represent

\begin{tabular}{|c|c|c|c|c|}
\hline & Num & $\begin{array}{l}\text { er of Attr } \\
\text { oring Weig }\end{array}$ & $\begin{array}{l}\text { outes } \\
\text { at) }\end{array}$ & \\
\hline Category & $\begin{array}{l}\text { High } \\
(100)\end{array}$ & $\begin{array}{l}\text { Medium } \\
\quad(10)\end{array}$ & $\begin{array}{l}\text { Low } \\
(1)\end{array}$ & $\begin{array}{c}\text { Child Attribute Values } \\
\text { (Family Preference Values) }\end{array}$ \\
\hline \multicolumn{5}{|l|}{ Child Demographics } \\
\hline Age & 1 & 0 & 0 & $\begin{array}{c}\text { Current age } \\
(\mathrm{Max} / \mathrm{min} \text { age })\end{array}$ \\
\hline Race/Ethnicity & 6 & 0 & 0 & $\begin{array}{l}\text { Applicable/not applicable } \\
\text { (Preferred/not preferred) }\end{array}$ \\
\hline Gender & 1 & 0 & 0 & $\begin{array}{c}\text { Male/female } \\
\text { (Male/female/either) }\end{array}$ \\
\hline \multicolumn{5}{|l|}{ Child Status } \\
\hline Educational Status & 0 & 1 & 0 & $\begin{array}{l}\text { Applicable/not applicable } \\
\text { (Approved/not approved) }\end{array}$ \\
\hline Special Needs & 6 & 7 & 0 & $\begin{array}{l}\text { Applicable/not applicable } \\
\text { (Approved/not approved) }\end{array}$ \\
\hline \multicolumn{5}{|l|}{ Characteristics of Child } \\
\hline Health & 0 & 3 & 7 & $\begin{array}{c}\text { Yes/no/unknown } \\
\text { (Acceptable/will consider/unacceptable) }\end{array}$ \\
\hline Education & 0 & 1 & 7 & $\begin{array}{c}\text { Yes/no/unknown } \\
\text { (Acceptable/will consider/unacceptable) }\end{array}$ \\
\hline Characteristics and Behaviors & 5 & 5 & 11 & $\begin{array}{c}\text { Yes/no/unknown } \\
\text { (Acceptable/will consider/unacceptable) }\end{array}$ \\
\hline Connections and History & 0 & 0 & 14 & $\begin{array}{c}\text { Yes/no/unknown } \\
\text { (Acceptable/will consider/unacceptable) }\end{array}$ \\
\hline Contact with Birth Family & 0 & 0 & 1 & $\begin{array}{c}\text { Yes/no/unknown } \\
\text { (Acceptable/will consider/unacceptable) }\end{array}$ \\
\hline
\end{tabular}

\footnotetext{
Note: Items in italics indicate child attribute values for which the attribute does not count as part of the total
} matching score.

have led to their exclusion from the final model for predicting child outcomes, and we do not make a recommendation about whether to reduce the emphasis on these child characteristics.

\section{Match Assessment Tool}

PAE is only one of several governmental and non-profit institutions that have developed tools to assess a possible match between a child and family. Hanna and McRoy (2011) describe the practice of matching in adoption as a means of finding families that have the right capabilities for handling a child's special needs and identifying gaps in a family's capabilities. They emphasize the need for standardization and data collection, point to match assessment tools as an important part of the family finding process, and review seven different tools used in practice by public and private agencies. Although some tools use more attributes (up to 277) than PAE, the design of PAE's existing matching tool surpasses all seven tools in terms of nuance in how attributes are weighted and its ambitions for helping to find families within a statewide network. 


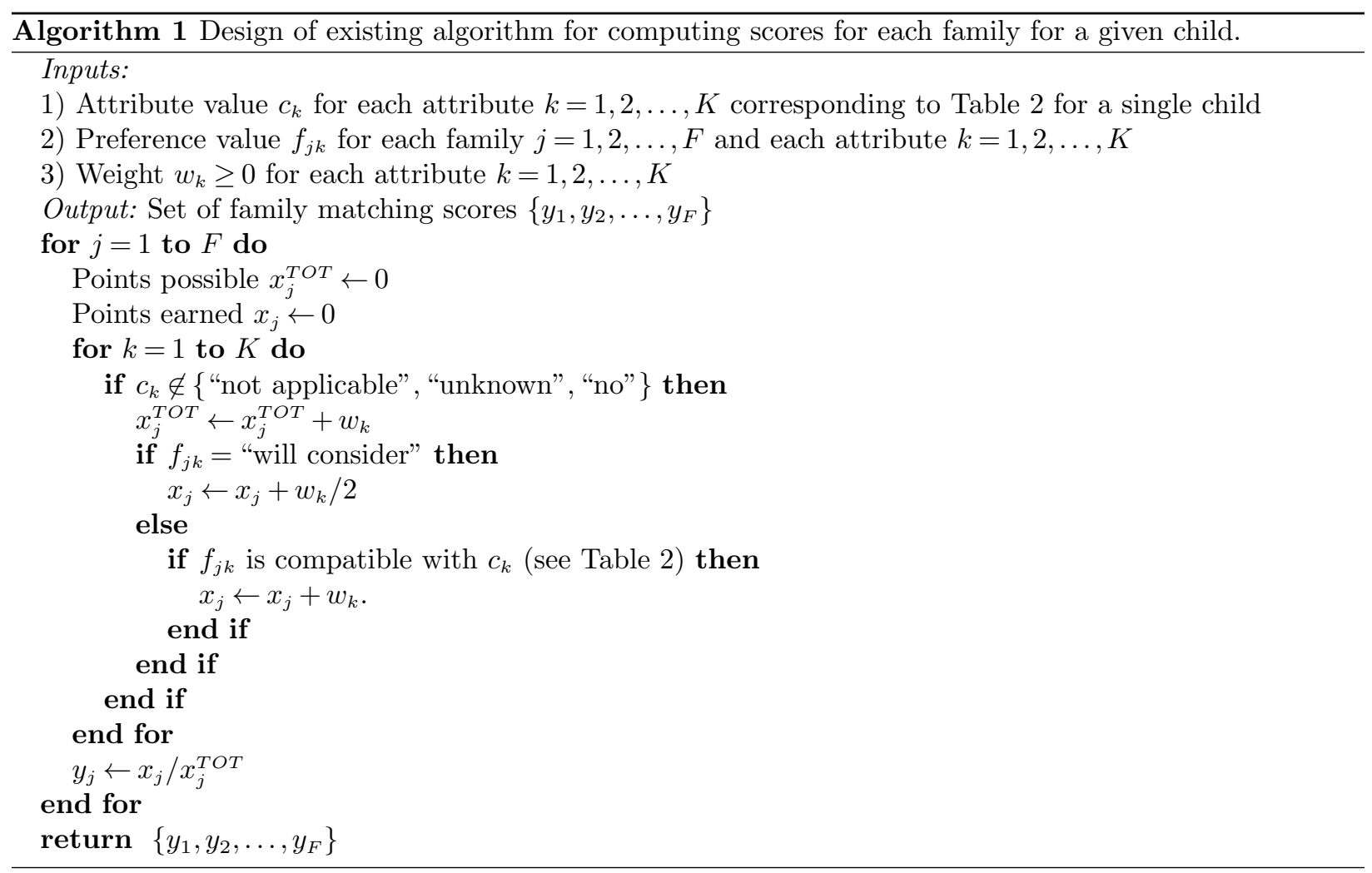

According to its intended design — which we formally express in Algorithm 1 - PAE's match assessment tool computes a family's score between $0 \%$ and $100 \%$ for a child based on 78 pairs of child attribute values and family preferences from CY 130 and CY 131 registration forms (Table 2). PAE managers had assigned a number of possible points to each of these pairs, which is 100 for items of high importance, 10 for items of medium importance, and 1 for items of low importance. When a special need or other attribute is not applicable or of an unknown state for a child, no points are either eligible or awarded to each family for that attribute. We note that PAE also does not give preference to families who say that a special need is undesirable when matching a child who does not have that special need. This practice could create incentives for families to hide special needs that they can accommodate if the families want to be considered for children without those special needs. For attributes that are applicable for a child, a positive family response — a child's age within the family's range, a matching gender, and answers of preferred, approved, or acceptable — receives all possible points for the item. An answer of "will consider" receives $50 \%$ of the possible points. Otherwise, the family's answer receives no points. For a specific child, the family's score is simply the sum of points received for their answers divided by the sum of possible points for the child. The "Matching Tool Spreadsheet" section of the appendix provides an example match score for a child and family. 
In recent years, PAE has struggled to make match recommendations that help case workers to find and assess families. Specifically, coordinating algorithm design with an information technology contractor and managing data collected over time across Pennsylvania's 67 counties proved difficult for PAE, and child case workers received unhelpful or illogical match recommendations as a result of a flawed implementation of Algorithm 1. The automated match recommendations were even abandoned for a period of over two years during which PAE coordinators manually searched through CY 130 and CY 131 forms to provide match suggestions.

Recognizing the shortcomings of the current rules for choosing matches, we worked with the SWAN managers to redesign the matching tool. This resulted in a spreadsheet-based algorithm that use PAE data about families and children to select matches. For ease of implementation, we focused on policies that had the same form as the PAE's match assessment tool. In particular, we considered policies that are based on a point system and ranked families according to some compatibility criteria. Rather than making specific assumptions about the relative importance of each criteria, our method offers the PAE managers the flexibility to select their desired weights and any other geographical constraints, as shown in Figure A.1. To find select prospective families for a child, the spreadsheet tool computes a ranked list of families for a child using CY 130 and CY 131 information stored in tables elsewhere in the matching tool.

The matching algorithm that we implemented differs in two aspects from those that have been applied in prominent centralized two-sided matching applications. First, the algorithms studied in that literature, such as Gale and Shapley's deferred acceptance algorithm, produce a batch of "final" matches that are concurrently implemented. However, our algorithm generates a list of mere recommendations, which may be implemented in conjunction with the judgment of the professionals. In this sense, our approach is closer to that adopted by the literature on semi-decentralized matching platforms such as those for online dating and job matching. The literature on these markets almost exclusively focuses on estimating participants preferences as opposed to increasing match success rate and quality, which we pursue here. The second, more subtle difference is that in centralized matching, participants are required to rank-list all available options, whereas here such ranking information is impossible to directly elicit because of limitations such as market size and informational asymmetry. One main function of the above algorithm can be seen as constructing such preferences from given pieces of information in the CY 130 and CY 131 forms and using them as the basis of recommendations. 


\section{Simulation of the Pennsylvania Adoption Exchange}

To examine the impact of a simple matching tool's effective use on the network's overall adoption rate, we represent PAE's matching process as a discrete-event simulation. We show the value of a statewide pool of families compared to a decentralized search process, and analyze how the ability of PAE to predict a match's success increases the number of matches. Specifically, we model how different levels of information about child attributes and family preferences affect the number of matches and the number of attempts before a successful match. We rely on the results of the regression analysis from the previous section to identify the most important child attributes for the simulation and additionally introduce relevant family registration data to model family preferences.

As an alternative to conventional techniques such as clinical trials, which would require many years to evaluate, discrete-event simulation has long been used to estimate the effects of policy changes, especially organ allocation policies (cf. Ata et al. (2012)). Similarly, our discrete-event simulation model of PAE's operation is modular and based on input parameters estimated with real data. Some of the simulation studies on organ allocation use a finite-horizon model, which is necessary since the data is highly time-dependent and reaching steady-state is very unlikely unless there is an alternative therapy for transplant patients. However, we assume stationary parameters in our simulation model, which is justified in the context of child adoption as the population characteristics of children and families in the system do not change dramatically over time.

The adoption network is divided into regions that constitute separate adoption networks defined by geographical and/or institutional barriers. Children may only be adopted by families that reside within the same region. Decreasing the number of regions to increase the size of each region provides each separate network with more prospective families and more children to match. We model the state of the PAE before our project as 20 separate regions due to the ineffectiveness of the central matchmaker. In that case, each region may correspond to a large county or a coalition of smaller counties in Pennsylvania. Because county case workers do face geographical limitations in matching, we do not expect a perfect centralized matchmaker to operate as a single region. Instead, managerial insights are primarily motivated by two cases: doubling the region size - i.e., dividing the state into 10 regions instead of 20 regions - and a system with four regions corresponding to PAE coordinators who provide match recommendations to county case workers. 

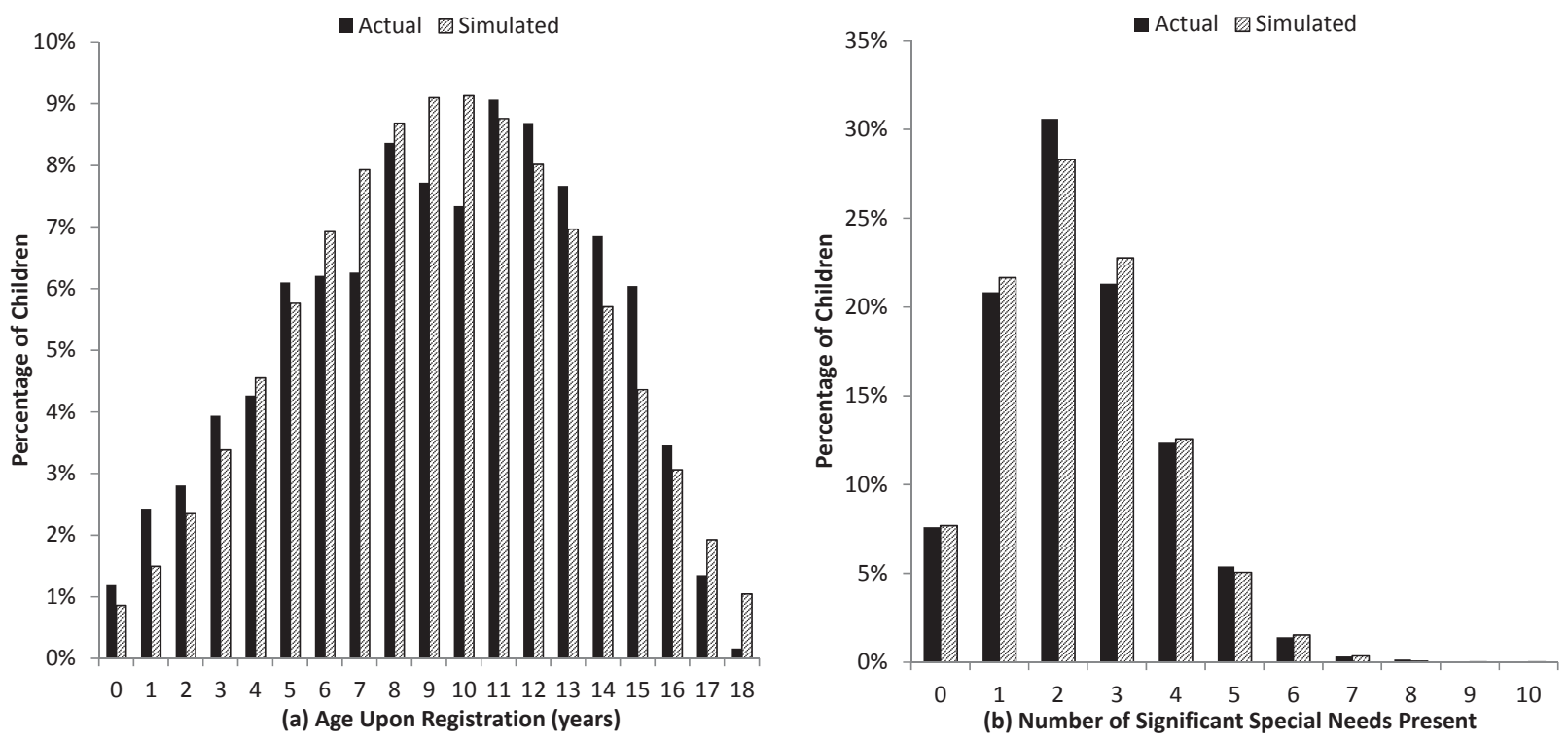

Figure 1 Based on age upon registration data for 1,853 children, we simulate the child's age using a beta distribution and use a binomial random variable to simulate the number of significant special needs present for each child.

\section{Children}

Children are defined by their age, number of special needs, and region in which they reside. A younger child is generally preferred to an older child, and a child with fewer special needs is generally preferable to a child with more special needs. The age attribute corresponds to the child's age upon registration with PAE. Using available data for the 1,853 children who have been registered with PAE, we fit the data using the input analyzer tool of @Risk and compared alternatives using a q-q chart. A beta distribution with shape parameters $\hat{\alpha}=5.7736$ and $\hat{\beta}=4.8877$ and scaled to be within the interval $[-5.4648,22.738]$ was determined to produce the best fit (Figure 1a). In the simulation, any age values outside the interval $[0.0,19.0]$ were discarded and resampled.

The special needs attribute corresponds to a count of the presenting attributes out of the ten child attributes that had a significant negative coefficient with a value less than -0.05 in the OLS regression analysis. This cutoff is arbitrary and used only in the simulation analysis to designate attributes of high importance. As with age, we fit the data using @Risk's input analyzer, and modeled the number of special needs present as a binomial random variable with parameters $n=14$ and $p=0.16741$ (Figure 1b). Any special needs values greater than 10 were discarded and resampled. 

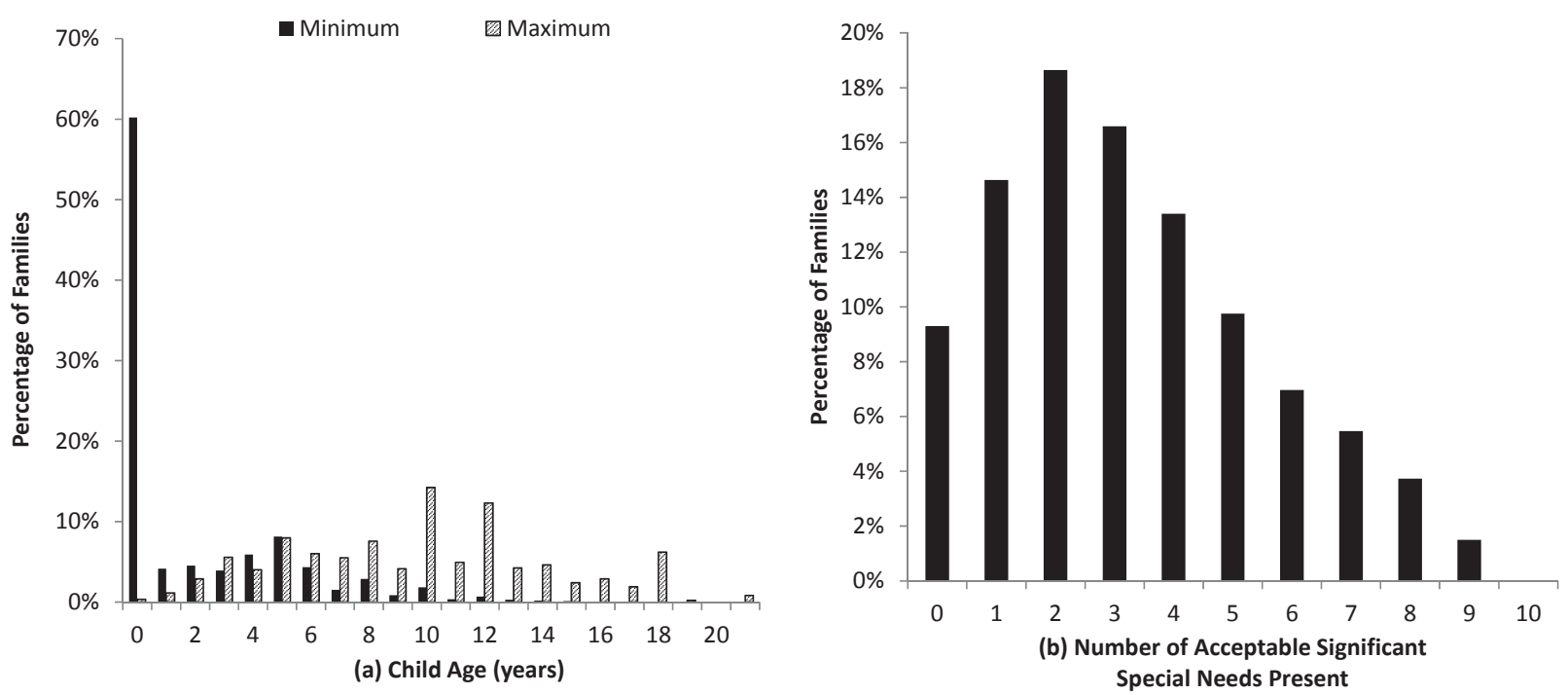

Figure 2 To simulate family preferences, we sample actual preferences from 2,194 registered families.

The registration age and number of significant negative special needs are positively correlated with a correlation coefficient of 0.230 . Therefore, we used a Normal-To-Anything (NORTA) process with two base vectors that have a correlation coefficient of 0.239 , which was obtained via a simulation approach. The standard multivariate normal vectors that follow a NORTA distribution are transformed to the age and special needs distributions using the method as described by Biller and Ghosh (2006).

The value of the child's region attribute is a random variable uniformly distributed over all regions. Children arrive in the system as a Poisson process with a rate of 239 per year, which is the average number of children who were fully registered with PAE annually between 2007 and 2012 .

\section{Families}

Families are defined by their region of residence and their preferences for an adoptive child's age and maximum number of special needs, as well as the relative weight of the age preference compared to the special needs preference. They arrive to the matching system as a Poisson process with rate 282 per year, which is the mean number of families to fully register with PAE as approved adoption resources each year between 2007 and 2012. Managers estimate that there are in the order of 1,000 prospective families available at any point in time, which implies that the expected time in system is 3.55 years by Little's Law. Thus, we model the family's time in the system as an exponential random variable with a mean of 3.55 years, as the distribution of families' time in system is not tracked by PAE. We note that higher number of families compared to the 
number of children in the system creates a disparity in the distribution of children available to adopt and the distribution of family preferences, which is reflected in the PAE system through children who "age out" of the system without an adoptive placement.

We model the families' behavior as myopic, accepting the first child that they are offered for which their utility of a match with the child is positive. The model behind the family acceptance decision is discussed in the "Matching Model and Simulation Details" section of the appendix. Once a family accepts a child, the family departs from the system. The values for a child's minimum age, maximum age, and number of acceptable special needs are sampled together from the data on 2,194 families (Figure 2). As with children, families are uniformly distributed over the regions.

\section{Matching Process}

We represent the matching process as a series of events that take place upon the arrival of a child in the system, which is viewed as the driver of the matching process by PAE managers. Matches are offered sequentially to families within the child's region. In each system, families are sorted according to criteria that correspond to PAE's operation with different levels of information. The highest-ranking family is selected and offered the child as a match. If a family accepts a match (i.e., its utility for the match is positive), both the family and child depart from the system. Whether a family's utility is positive depends on the child's characteristics, the family's preferences, and a random term to represent the uncertainty of attraction. Because data is not available to estimate the randomness of this process, we tested two values of the variability of the error term that we label as low attraction variability and high attraction variability. If the family rejects the match, the family remains in the system and another match - up to ten total match attempts is attempted for the child. For simplicity, we model the matching process as an instantaneous event, although in practice time elapses between sequential matching attempts. If no match is found for the child, the child departs from the system. A flowchart representing this process is provided as Figure A.3 in the "Matching Model and Simulation Details" section of the appendix.

We present three methods for ranking families to investigate the value of information. The three methods and their interpretations are as follows:

1. Critical Attribute (CA) represents a system in which case workers can search for families based on either the age or special needs attribute due to constraints on their search time and effort. We represent the matching process before our collaboration with PAE as following the CA policy. 


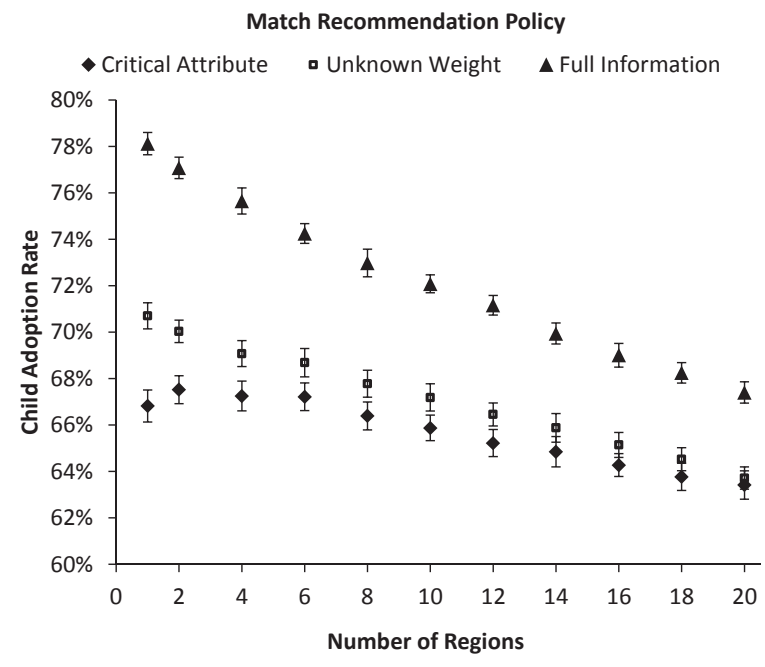

(a) Low Attraction Variability

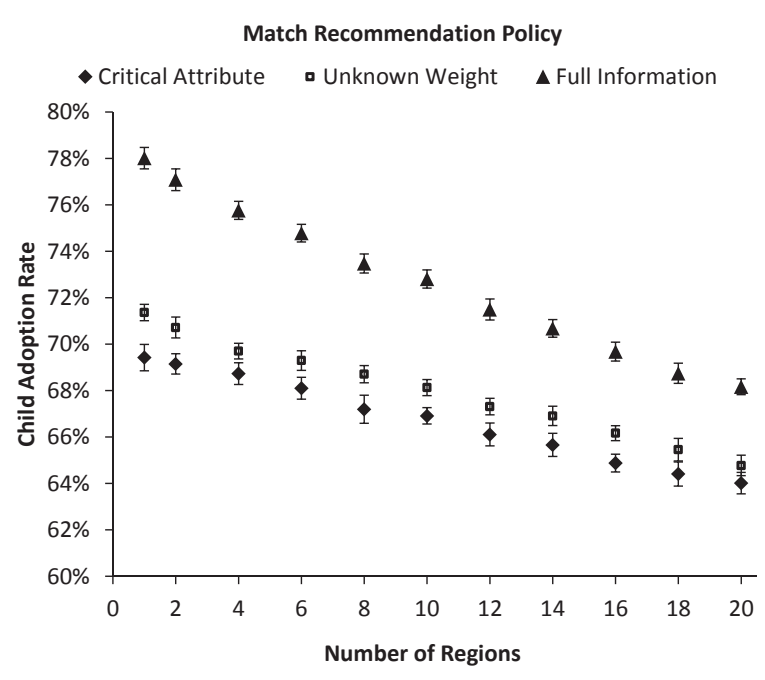

(b) High Attraction Variability

Figure 3 The child adoption rate increases with the quality of information available for matching and decreases with the number of regions (i.e., the segmentation of the network). Bands represent $95 \%$ confidence intervals.

2. Unknown Weight (UW) represents a simple version of a centralized matching system that is limited in its ability to properly incorporate family preference information. Age and special needs attributes are given equal weight in this model.

3. Full Information (FI) represents an improved version of PAE's centralized matching system with families sorted based on a known age preference, special needs preference, and preference weighting term.

\section{Simulation Results}

We first compare the simulated mean percentage of children matched over the five-year horizon based on the attraction variability and number of regions for CA, UW, and FI decision rules. The adoption rate increases with the amount of information about the families' preferences utilized in the match recommendation process (Figure 3); i.e., CA exhibits a lower adoption rate than UW, which in turn has a rate lower than that of FI. The UW policy only slightly improves upon the CA policy with a maximum increase of 3.9 percentage points. However, the FI policy improves on the CA policy by between 4.0 and 11.3 percentage points in the child adoption rate. Whether the attraction variability is low or high appears to have minimal impact on the performance of the policies in terms of the overall adoption rate.

The mean adoption rate always either increases as the number of regions decreases or shows a statistically insignificant decrease. A completely centralized system (i.e., one region) results in an adoption rate that is 
between 3.4 and 10.7 percentage points higher - depending on the attraction variability and the recommendation rule - than the completely decentralized case with 20 regions. This validates the role of a statewide network. With a larger pool of families, it is more likely for a family to exist that seeks the type of child being matched or can accommodate the child's special needs.

In addition to an increase in the adoption rate for the UW and FI policies compared to the CA policy, the better use of information also corresponds to a decrease in the mean number of match attempts until a successful adoption for children who are successfully adopted (Figure 4). We study this metric as a proxy for two important secondary measures of success for the adoption network: the workload for case workers and time in system for the child. Fewer attempts until success means less work for overburdened case workers and a shorter time in state custody for a child. Depending on the number of regions, the UW and FI policies cause the mean number of match attempts until success to decrease between $32 \%$ and $41 \%$ when attraction variability is low and between $17 \%$ and $21 \%$ when attraction variability is high.

We further investigate the effect of the attraction variability, which represents the unpredictability of attraction between an individual family and child. When the attraction variability is high, match success is inherently more difficult to predict, which results in an increase in the mean number of attempts per successful adoption of up to 0.81 (Figure 4). Comparing the change in the mean adoption rate as the number of regions decreases, the difference between the low and high attraction variability cases (in relation to the calibration point) is almost always less than $1 \%$. This indicates that the underlying match unpredictability has relatively little impact on the mean adoption rate compared to the matching rule and number of regions. The only exceptions are for the CA policy with 1 or 2 regions when high attraction variability results in an adoption rate that is 1.0-2.0 percentage points higher than the adoption rate when the attraction variability is low. In these cases, the lower match success predictive power of the CA policy for ranking families, the high attraction variability, and the small number of regions means that it is relatively more likely for families who are offered children later in the sequence of ten offers to be stronger candidates.

These results justify the value of a statewide adoption network and show that the quality of information about family preferences is critical to its success. Furthermore, we have shown that better information improves secondary metrics of system performance that can be interpreted as reducing case workers' workloads and time in system for children. If the adoption network can even just double the size of its regions so that the number of regions is 10 instead of 20 while improving how it elicits family preferences for matching 


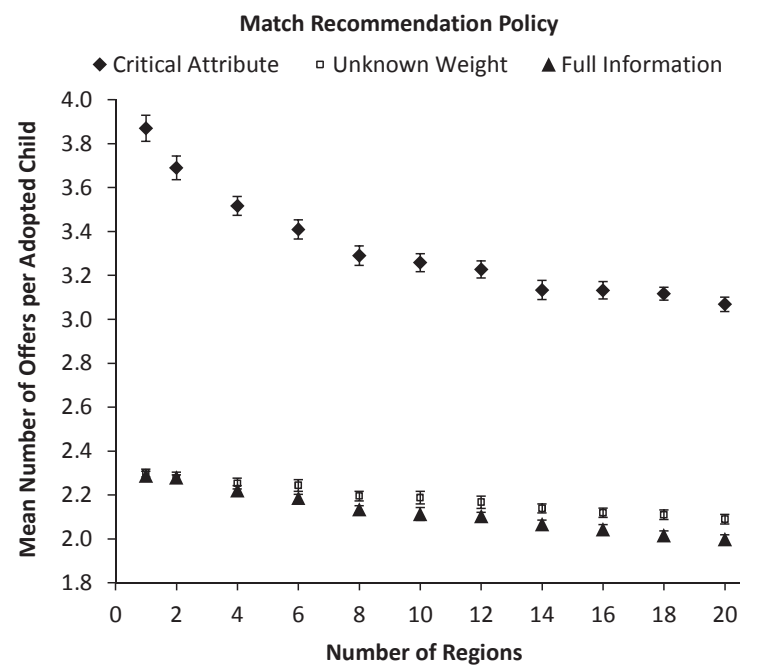

(a) Low Attraction Variability

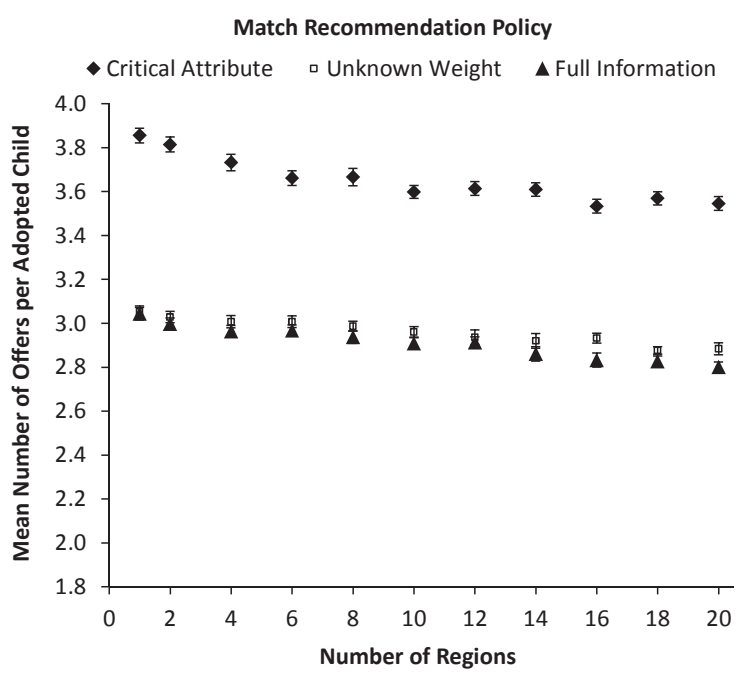

(b) High Attraction Variability

Figure 4 Improving the information available for matching reduces the average number of attempts before a successful adoption. Bands represent $95 \%$ confidence intervals.

(i.e., follow the FI policy), the number of successfully adopted children can increase by approximately 21 children per year. Additional results and managerial insights related to improvements in match quality are discussed in the "Matching Model and Simulation Details" section of the appendix.

\section{Process Improvements and Results}

To achieve the possible improvement in the adoption rate demonstrated in the simulation, we worked with PAE to improve the information it collects and its family ranking tool for matching. In this section, we discuss these changes, as well as the incentives that affect how participants reveal their preferences. A spreadsheet matching tool in use by PAE coordinators provides the most tangible evidence of improvements from our collaboration. While using a computerized tool for matching is not unique to our project, we are the first to connect the quality of preference information to the overall network adoption rate and show how underlying incentives for how families reveal their preferences can diminish the usefulness of recommendations. We also suggest improvements to the family ranking algorithm that are novel to the practice of matching in child adoptions.

\section{Registration Information}

Through our interviews with child case workers and discussion of the simulation results, PAE managers came to recognize new potential for gathering information during the registration process as a driver of the 
overall network adoption rate. Specifically, our research collaboration has led to the collection of additional information to use in making match recommendations. Although PAE managers believed revising the CY 130 and CY 131 forms to be an arduous process, especially since the forms had been recently revised, they are beginning to collect child and family information through an online survey format with a new set of questions. Their intuition about data that would be most valuable for predicting matches informed their selection of these new questions. In particular, these new questions focus on the child's positive attributes, such as interests or hobbies, that might predict attraction between children and families. Other questions focus on family attributes that could be compared to child or child case worker preferences for families without certain pets or other children in certain age ranges. The questions have received approval from the state for use by PAE and are in the process of being implemented.

Furthermore, as a result of our project, PAE managers have begun tracking the results of match recommendations for future analysis. While the set of information used for match recommendations is currently based on managerial intuition, we have encouraged PAE to maintain data about match attempts and their results in order to scientifically evaluate the power of different questions to predict matches. Econometric analysis of child attributes, family preferences, and results of match attempts would allow PAE managers to better estimate the probability of success of a child-family match and assess which questions are more or less important in predicting a match.

\section{Spreadsheet Matching Tool}

After several design and feedback iterations, PAE coordinators have begun to use our matching tool prototype to suggest families to county-level case workers. The matching tool prototype has also allowed them to gain insights into the matching rules and to begin to think about what matching rules help produce the best matches for children. We have supported the addition of features, such as geographical preferences for families, to improve the tool's value to PAE managers.

Compared to other matching tools discussed in Hanna and McRoy (2011), we use the same underlying framework of linearly weighted questions to score a family's suitability for a child, and add three simple innovations. First, the user can directly specify the weights for each attribute to help determine which attributes are most important for selecting a family for a child. PAE managers and coordinators observed that children are "labelled" by certain special needs (e.g., fire starter, animal abuser) when the underlying behavior that prompted the label was viewed as innocuous. They felt having the ability to adjust matching 
tools weights based on knowledge of the severity of a child's special needs could produce better matches. This feature also allowed the easy change of default weights for factors identified as important in the regression analysis. Second, the user can state geographical preferences for the family's county of residence, which can be important in assessing the feasibility of a match if continuing community or familial relationships is important for children. Finally, as noted in Hanna and McRoy (2011), social workers who assist families can use the tool to identify shortcomings in the family's capabilities for a child and prepare appropriate support mechanisms. To this end, we have added score summaries by category so that users can more easily identify a family's strengths and challenges, as well as output reports for use by the matching committee that show how the child and family compare for each attribute.

\section{Information Incentives}

We also examined how families and child case workers interact with PAE to understand intentional or unintentional behavior patterns that may reduce the matching process's effectiveness. Through conversations with PAE managers and preliminary runs of the matching system, we noticed that the system is vulnerable to strategic manipulation by families in how they complete their CY 131 forms. Because rejecting a child is very easy for families - a telephone hotline is available to review details of and accept/reject an available child for which the family is recommended - families have an incentive to overstate their willingness to accept children with special needs. This allows a family to gain additional information and be considered for more children. Furthermore, this behavior does not necessarily result from conscious manipulation of the PAE system; different families may be inherently more or less strict in how they interpret the difference between an "acceptable" and "will consider" response to a specific special need. The current system gives families the incentive to err on the side of choosing "accept," which makes differentiating between families more difficult for PAE.

We recommended a process change and an algorithmic feature to overcome the challenge of families' overstating their tolerance for children with special needs. First, we recommended that matching occur in small batches so that PAE coordinators - who use the matching tool - can observe if families are chosen too frequently and further investigate the appropriateness of those families as recommended matches. PAE managers initially welcomed this suggestion, and decided that monthly matching meetings would work well with the adoption framework. They also augmented it based on their experiences with a rule that PAE coordinators should wait 30 days between successive recommendations of the same family. Second, the 
family-child match score was adjusted for three criteria — race, age, and gender — to reward families whose preferences more closely fit the child's attributes. For example, a family who indicates a preference of male or female receives a higher score on the gender attribute than a family who indicates a preference of "either" if the child is of the preferred gender.

Using a matchmaking experiment, we show that the rewarding of narrow preferences more effectively spreads the recommendations over the pool of families. For each active child, we calculated the top five matches (plus ties) from the list of active families using a scoresheet with and without rewards for narrow preferences over age, race, and gender. Without rewards for narrow preferences, we noticed that only $7.7 \%$ of families received at least one match, although we should note that this number would increase in practice when geographical preferences are applied. With rewards for narrow preferences, the number of families who received at least one match increased by $41 \%$. We proposed further rewarding narrow preferences based on the number of child special needs that the family states are acceptable, based on the acceptable number of all special needs or the ten special needs identified as significant in the regression analysis.

Another difficulty that PAE managers emphasized is placement decision making by child case workers. SWAN managers and even case workers themselves indicate that some case workers also struggle with emotional attachment to children to an extent that dispassionately making a placement decision according to the child's best interests can be very challenging. In other words, some case workers' emotional attachment can cause them to hold out for the "perfect" family, when another family likely to be suitable for the child is available. PAE managers have found the spreadsheet matching tool to be valuable as a mechanism to enforce the conceptualization of trade-offs. In conversations between case workers and PAE coordinators who use the matching tool, observing that no family is likely to be a $100 \%$ match can lead to discussions about the strengths and weaknesses of a family-child match.

\section{Conclusions}

In the collaboration described in this paper, we helped PAE improve the processes for recommending prospective families for children in state custody. We believe that these changes increase PAE's value to case workers in their efforts to find find families, and will increase the percentage of children who find permanent placements. Furthermore, PAE has begun collecting additional data about the matching process to scientifically compare families' stated preferences to their actual decisions. This will enable future work that would analyze the matching weights and relative value of the registration questions. The challenge of making match 
recommendations in a two-sided matching market certainly extends beyond the adoption of children in state custody, and insights from this paper may apply to matching for foster care placements.

\section{Acknowledgments}

The authors wish to thank Jane Johnston, Pennsylvania Adoption Exchange Division Manager, and the managers and staff of the Pennsylvania Statewide Adoption and Permanency Network for their extensive support of this project. The second author acknowledges financial support through NSF Grants CMMI 1351821 and CMMI 1334194 and the Berkman Faculty Development Fund.

\section{Appendix}

\section{Matching Tool Spreadsheet}

To illustrate the match scoring rule used in the matching tool, we provide an example that corresponds to Figure A.1 and Figure A.2. Based on the child's presenting needs, a total of 425 points are possible for any family matched to the child, including 100 for age, 100 for race, 100 for gender, 60 for special needs, and 65 for characteristics. The family receives 100 points because the child is within the family's stated minimum and maximum ages, 100 points because the family's preference for a white child matches the child's race, and 100 points as the family stated that either gender was acceptable. The family receives 40 out of 60 possible points for the child's special needs, losing points as the family is not approved to adopt a child with "drug exposed infant" and "abuse history" designations. For child characteristics, the family is allotted 44 of 65 possible points for a total of 384 out of 425 possible points, which translates to a score of $90.35 \%$.

\section{Matching Model and Simulation Details}

Children are defined by a type $c=\{a, s, r\}$, which reflects that child's desirability on two attributes — age $a \in[0,19]$ years and number of significantly negative special needs $s \in\{0, \ldots, 10\}$ - and a residence region $r \in\{1, \ldots, R\}$, where $R$ is the total number of regions.

Families are defined by their type $f=\left\{a_{M I N}, a_{M A X}, s^{\prime}, r, \alpha\right\}$, which represents their range of acceptable ages $\left[a_{M I N}, a_{M A X}\right]$ with $a_{M I N}, a_{M A X} \in\{0, \ldots, 19\}$ and $a_{M I N}<a_{M A X}$, their tolerance for child special needs $s^{\prime} \in\{0, \ldots, 10\}$, a weight $\alpha \in[0,1]$ to express the relative importance of age and special needs, and a region attribute $r \in\{1, \ldots, R\}$. We also define a utility function for the purpose of indicating whether a family will accept an offered child. A child age component and a child special needs component comprise the utility function, and their relative weight is dictated by the weighting term $\alpha$. With only limited information about 
Match Scoring Tool for Ranking Families

\begin{tabular}{|c|c|c|c|c|c|}
\hline & \multirow{2}{*}{$\begin{array}{l}\text { (Fictitious) } \\
\text { (Fictitious) }\end{array}$} & \multirow[b]{4}{*}{ Child Info } & \multirow[b]{4}{*}{ Family Pref } & \multicolumn{2}{|c|}{ Points } \\
\hline Family ID & & & & $\begin{array}{r}\text { Earned } \\
384 \\
\end{array}$ & $\begin{array}{r}\text { Possible } \\
425 \\
\end{array}$ \\
\hline Score & $90.35 \%$ & & & \multirow{3}{*}{ Points } & \multirow{3}{*}{ Pts Possible } \\
\hline \multirow[t]{2}{*}{ Weight } & & & & & \\
\hline & DEMOGRAPHIC INFORMATION & & & & \\
\hline \multirow[t]{3}{*}{100} & Age & 13 & & 100 & 100 \\
\hline & Low Age & & 10 & & \\
\hline & High Age & & 14 & & \\
\hline \multicolumn{2}{|c|}{100 Race/Ethnicity } & & & & \\
\hline \multirow{7}{*}{\multicolumn{2}{|c|}{$\begin{array}{l}\text { African American } \\
\text { Hispanic } \\
\text { White } \\
\text { American Indian/Alaskan Native } \\
\text { Asian } \\
\text { Native Hawaiian/Other Pacific Islander } \\
\text { Child Gender }\end{array}$}} & NA & Not Preferred & 0 & 0 \\
\hline & & NA & Not Preferred & 0 & 0 \\
\hline & & Applicable & Preferred & 100 & 100 \\
\hline & & $\mathrm{NA}$ & Not Preferred & 0 & 0 \\
\hline & & NA & Not Preferred & 0 & 0 \\
\hline & & $\mathrm{NA}$ & Not Preferred & 0 & 0 \\
\hline & & Female & Either & 100 & 100 \\
\hline \multicolumn{2}{|r|}{ SPECIAL NEEDS INFORMATION } & & & & \\
\hline \multicolumn{2}{|c|}{$\begin{array}{l}10 \text { Drug Exposed Infant } \\
10 \text { Emotional Disability }\end{array}$} & Applicable & Not Approved & 0 & 10 \\
\hline \multirow{3}{*}{$\begin{array}{r}10 \mathrm{E} \\
100 \mathrm{H} \\
10 \mathrm{M}\end{array}$} & Emotional Disability & Applicable & Approved & 10 & 10 \\
\hline & HIV & NA & Not Approved & 0 & 0 \\
\hline & MH Diagnosis & NA & Approved & 0 & 0 \\
\hline \multirow{2}{*}{$\begin{array}{r}100 \\
10\end{array}$} & MR Diagnosis & NA & Not Approved & 0 & 0 \\
\hline & Multiple Placement History & Applicable & Approved & 10 & 10 \\
\hline \multirow{2}{*}{$\begin{array}{r}100 \\
10\end{array}$} & Physical Disability & NA & Approved & 0 & 0 \\
\hline & Runaway History & NA & Approved & 0 & 0 \\
\hline \multirow{2}{*}{$\begin{array}{l}100 \\
100\end{array}$} & Sexual Abuse History & NA & Approved & 0 & 0 \\
\hline & Siblings & NA & Approved & 0 & 0 \\
\hline \multirow{2}{*}{$\begin{array}{r}10 \\
100\end{array}$} & Special Education Student & Applicable & Approved & 10 & 10 \\
\hline & Special Medical Care & NA & Approved & 0 & 0 \\
\hline \multirow{2}{*}{$\begin{array}{l}10 \\
10\end{array}$} & Abuse History & Applicable & Not Approved & 0 & 10 \\
\hline & Neglect History & Applicable & Approved & 10 & 10 \\
\hline
\end{tabular}

Figure A.1 PAE regional coordinators use a spreadsheet with customizable attribute weights that automatically computes scores for all families for a given child. ("NA" refers to an attribute that is not applicable for a child.)

family preferences, we use a uniform distribution for the weighting term, as justified for preference modeling with limited information by Kennan (2006).

We define a family's utility for a match with a child of type $c$ as

$$
u(c ; f):=\alpha\left(u^{A G E}\left(a, a_{M A X}\right)\right)+(1-\alpha)\left(u^{S N}\left(s, s^{\prime}\right)\right)+\epsilon,
$$

where $\epsilon$ is an error term that represents the randomness of a child's attractiveness to a family. We let $\epsilon$ be an independent random variable that follows a normal distribution with mean $\mu$ and standard deviation $\sigma$. The term $\sigma$ represents families' variability in their attractiveness for individual children. Without data to connect families' stated preferences to their acceptance decisions, we consider cases of $\sigma=0.1$ and $\sigma=0.2$, 


\begin{tabular}{|c|c|c|c|c|c|}
\hline Weight & CHILD CHARACTERISTICS & Child Info & Family Pref & Points & Pts Possible \\
\hline \multirow{2}{*}{ (5) } & \multirow{2}{*}{$\begin{array}{l}\text { 1. Does child have significant health issues? } \\
2 . \text { Does child have allergies or asthma? (may require treatment) }\end{array}$} & No & Acceptable & & \\
\hline & & Yes & Acceptable & 1 & 1 \\
\hline 10 & & Yes & Acceptable & 10 & 10 \\
\hline \multirow{2}{*}{$\begin{array}{l}1 \\
1\end{array}$} & 4. Does child have speech problems? (may require treatment) & No & Acceptable & & \\
\hline & \multirow{2}{*}{ 6. Is child legally deaf? } & No & Acceptable & & \\
\hline \multirow{2}{*}{$\begin{array}{l}1 \\
1\end{array}$} & & No & Will Consider & & \\
\hline & 7. Does child have vision problems? (may require treatment) & No & Will Consider & & \\
\hline 10 & 8. Is child legally blind? & No & Unacceptable & & \\
\hline \multirow{2}{*}{$\begin{array}{l}1 \\
1\end{array}$} & 9. Does child have dental problems? (may require treatment) & No & Acceptable & & \\
\hline & 10. Does child have orthopedic problems (special shoes, braces, etc) & No & Acceptable & & \\
\hline 10 & 11. Does child have seizures? & No & Will Consider & & \\
\hline 1 & & No & Acceptable & & \\
\hline \multirow{2}{*}{$\begin{array}{l}1 \\
1\end{array}$} & 14. Does child achieve at grade level in regular classes? & Yes & Acceptable & 1 & 1 \\
\hline & 15. Does child achieve below grade level in regular classes? & No & Acceptable & & \\
\hline 1 & 16. Is child in special education classes? & No & Acceptable & & \\
\hline 1 & 17. Does child have a learning disability? & No & Acceptable & & \\
\hline 1 & 18. Does child need classes for the emotionally or behaviorally handicapped? & Yes & Acceptable & 1 & 1 \\
\hline 1 & 19. Does child need tutoring in one or more subjects? & No & Acceptable & & \\
\hline 10 & 20. Does child have serious behavior problems in school? & Yes & Will Consider & 5 & 10 \\
\hline 1 & 21. Is child generally quiet and shy? & No & Acceptable & & \\
\hline 1 & 22. Is child generally outgoing and noisy? & Yes & Acceptable & 1 & 1 \\
\hline 1 & 23. Does child have emotional issues that requires therapy? & Yes & Acceptable & 1 & 1 \\
\hline 1 & 24. Does child tend to reject father figures? & No & Will Consider & & \\
\hline 1 & 25. Does child tend to reject mother figures? & No & Will Consider & & \\
\hline 1 & 26. Does child have difficulty relating to others and relating to other children? & Yes & Acceptable & 1 & 1 \\
\hline 1 & 27. Does child frequently wet the bed? & No & Acceptable & & \\
\hline 1 & 28. Does child frequently soil him/herself? & No & Will Consider & & \\
\hline 100 & 29. Does child masturbate frequently or openly? & No & Unacceptable & & \\
\hline 1 & 30. Does child have poor social skills? & Yes & Acceptable & 1 & 1 \\
\hline 10 & 31. Does child have problem with lying? & Yes & Will Consider & 5 & 10 \\
\hline 10 & 32. Does child have problem with stealing? & No & Will Consider & & \\
\hline 10 & 33. Does child frequently start physical fights with other children? & Yes & Will Consider & 5 & 10 \\
\hline 100 & 34. Does child abuse animals? & No & Unacceptable & & \\
\hline 10 & 35. Is child destructive with clothing, toys, etc.? & Yes & Will Consider & 5 & 10 \\
\hline 10 & 36. Does child use foul or bad language? & No & Acceptable & & \\
\hline 1 & 37. Does child have frequent temper tantrums? & Yes & Acceptable & 1 & 1 \\
\hline 1 & 38. Does child have difficulty accepting and obeying rules? & Yes & Acceptable & 1 & 1 \\
\hline 100 & 39. Does child exhibit inappropriate sexual behavior? & No & Will Consider & & \\
\hline 100 & 40. Does child have a history of running away? & No & Will Consider & & \\
\hline 100 & 41. Does child have history of playing with matches, setting fires? & No & Unacceptable & & \\
\hline 1 & 42. Does child have strong ties to birth family? & Yes & Acceptable & 1 & 1 \\
\hline 1 & 43. Does child have strong ties to foster family? & No & Acceptable & & \\
\hline 1 & 44. Is continued contact with siblings desirable? & No & Acceptable & & \\
\hline 1 & 45. Does child have a previous adoption disruption? & No & Acceptable & & \\
\hline 1 & 46. Was child sexually abused? & No & Will Consider & & \\
\hline 1 & 48. Was child exposed to promiscuous sexual behavior? & No & Will Consider & & \\
\hline 1 & 49. Was child conceived by rape? & No & Will Consider & & \\
\hline 1 & 50. Was child conceived as a result of prostitution? & No & Unacceptable & & \\
\hline 1 & 51. Are one or both parents addicted to alcohol? & Yes & Will Consider & 0.5 & 1 \\
\hline 1 & 52. Are one or both parents dependent on substances other than alcohol? & Yes & Acceptable & 1 & 1 \\
\hline 1 & 53. Do one or both parents have a criminal record? & Yes & Acceptable & 1 & 1 \\
\hline 1 & 54. Are one or both parents mentally retarded? & No & Unacceptable & & \\
\hline 1 & 55. Do one or both parents have a mental illness? & Yes & Will Consider & 0.5 & 1 \\
\hline 1 & 56. Does agency lack information about one or both parents? & No & Acceptable & & \\
\hline 1 & 57. Is child in contact with birth parents? & Yes & Acceptable & 1 & 1 \\
\hline & 58. Is child in contact with siblings? & No & & & \\
\hline & 59. Is child in contact with extended birth family? & No & & & \\
\hline & 60. Is child in contact with former foster family? & No & & & \\
\hline
\end{tabular}

Figure A.2 The spreadsheet tool also includes a section for "Child Characteristics" information.

to which we refer as low attraction variability and high attraction variability, respectively. Given $\sigma, \mu$ then becomes a tuning parameter for the simulation. The value of $\epsilon$ is only revealed when a match is attempted between a family and a child.

Lacking data to directly estimate families' preferences, we instead rely on the analysis of factors related to child outcomes from the previous section to create a model for family preferences. Specifically, we use the 
resulting coefficients from a linear regression model based on the child's managerially weighted outcome as a response variable and factors of age (linear), age (quadratic), and the number of significant negative special needs. The resulting model is

$$
\text { Outcome }(c)=0.8356+0.0426 a-0.0045 a^{2}-0.0476 s
$$

for which the intercept and all three coefficients are significant at a $99.9 \%$ confidence level. We use these coefficients from this model to estimate the age and special needs components of the utility function. Given a child's age $a$ and a family's preferred minimum age $a_{M I N}$ and maximum age $a_{M A X}$, we define the age component of a family's utility for a child as

$$
u^{A G E}\left(a, a_{M I N}, a_{M A X}\right):=\left\{\begin{array}{ll}
0.0426\left(a-a_{M A X}\right)-0.0045\left(a^{2}-a_{M A X}^{2}\right) & \text { if } a \geq a_{M I N} \\
0 & \text { if } a<a_{M I N}
\end{array},\right.
$$

which represents the difference in the effect of age upon outcome between the child's age upon registration and the family's maximum preferred age. For a family that prefers a child between 0 and 12 years old, the age component of the utility function is 0.235 for a child of age 4, 0.190 for age 8 , and 0 for age 12 . For older children, the value is -0.149 for a child of age $14,-0.334$ for age 16 , and -0.554 for age 18 . We note that, due to the quadratic term, the utility component is not strictly decreasing in age for very young children, but we ignore this effect just for simulation purposes as children younger than 3 only represent about $6 \%$ of the population. In general, these children are not difficult to place and are not the focus of PAE. Similarly, the special needs component of the family's utility for a child is defined as

$$
u^{S N}\left(s, s^{\prime}\right):=-0.0476\left(s-s^{\prime}\right),
$$

which represents the difference in the effect of the number of significant negative special needs and the family's number of corresponding acceptable special needs.

With this model, we can more precisely define the three family ranking methods:

1. Critical Attribute (CA): If $0.0426 a-0.0045 a^{2}<-0.0476 s$, then families are sorted according to $u^{A G E}\left(a, a_{M I N}, a_{M A X}\right)$. Otherwise, families are sorted according to $u^{S N}\left(s, s^{\prime}\right)$.

2. Unknown Weight $(U W)$ : Families are sorted according to their nominal utilities, which disregards the error term, for the child with the two attributes equally weighted (i.e., $\alpha=0.5$ ) to represent $\alpha$ unknown. 
Collected Statistics:

1. Number of children matched and unmatched

2. Number of offers until match acceptance for successfully adopted children

3. Percentage of families for which child attribute values are above families' stated maximum for each attribute

4. Family nominal utility for each successful match

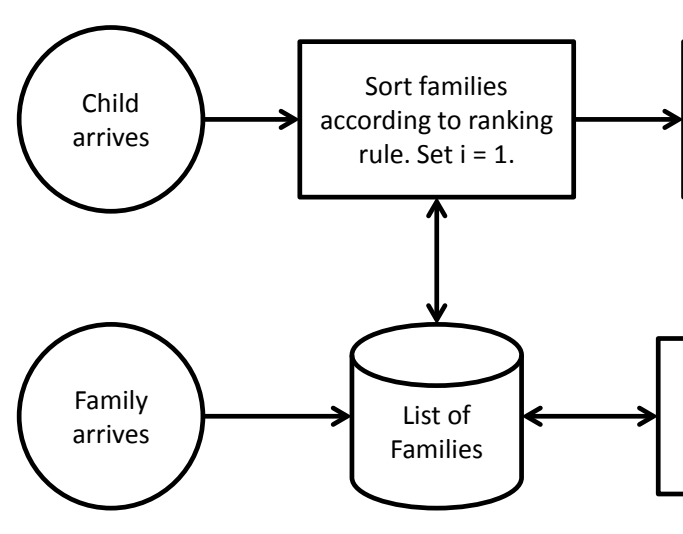

Family Characteristics

Minimum and maximum acceptable age, maximum number of acceptable significant negative special needs, weighting term for utility function, maximum waiting time before departure

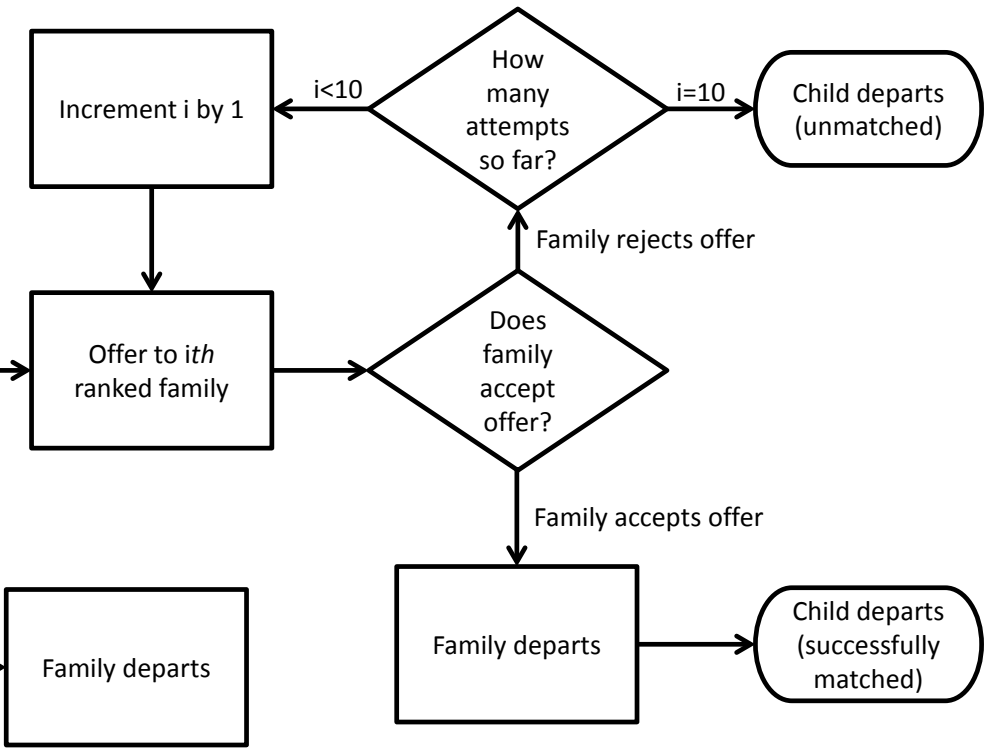

Child Characteristics:

Age, number of significant negative special needs

Figure A.3 When a child becomes available, we rank prospective families and sequentially make up to ten match attempts. A child is successfully adopted if at least one family accepts the child. Otherwise, the child is not adopted.

3. Full Information (FI): Families' types are known to the matchmaker, which given a child of type $c$ can rank the families according to their nominal utility $\alpha\left(u^{A G E}\left(a, a_{M A X}\right)\right)+(1-\alpha)\left(u^{S N}\left(s, s^{\prime}\right)\right)$.

The simulation is initialized by starting with a pool of 1,000 randomly generated families. The replication length is five years and is preceded by a one-year warm-up period. The family attraction variability tuning parameter is set to $\mu=-0.1875$ for low attraction variability $\sigma=0.1$ and $\mu=-0.315$ for high attraction variability $\sigma=0.2$, which corresponds to a $64 \%$ success rate for the Critical Attribute rule and matches the expected quality-adjusted outcome value for children in the PAE system between 2005 and 2013 . We calibrated the simulation using the $\mathrm{CA}$ decision rule to represent the process by which county case workers manually searched through families' records focusing on their suitability for a small subset of child attributes, which most accurately describes PAE's functioning before changes were implemented as part of our collaboration. For each scenario — defined by a matching policy and number of regions — we used 25 replications so that we are $95 \%$ confident that the resulting mean match rate is within $1 \%$ of the true mean match rate. 

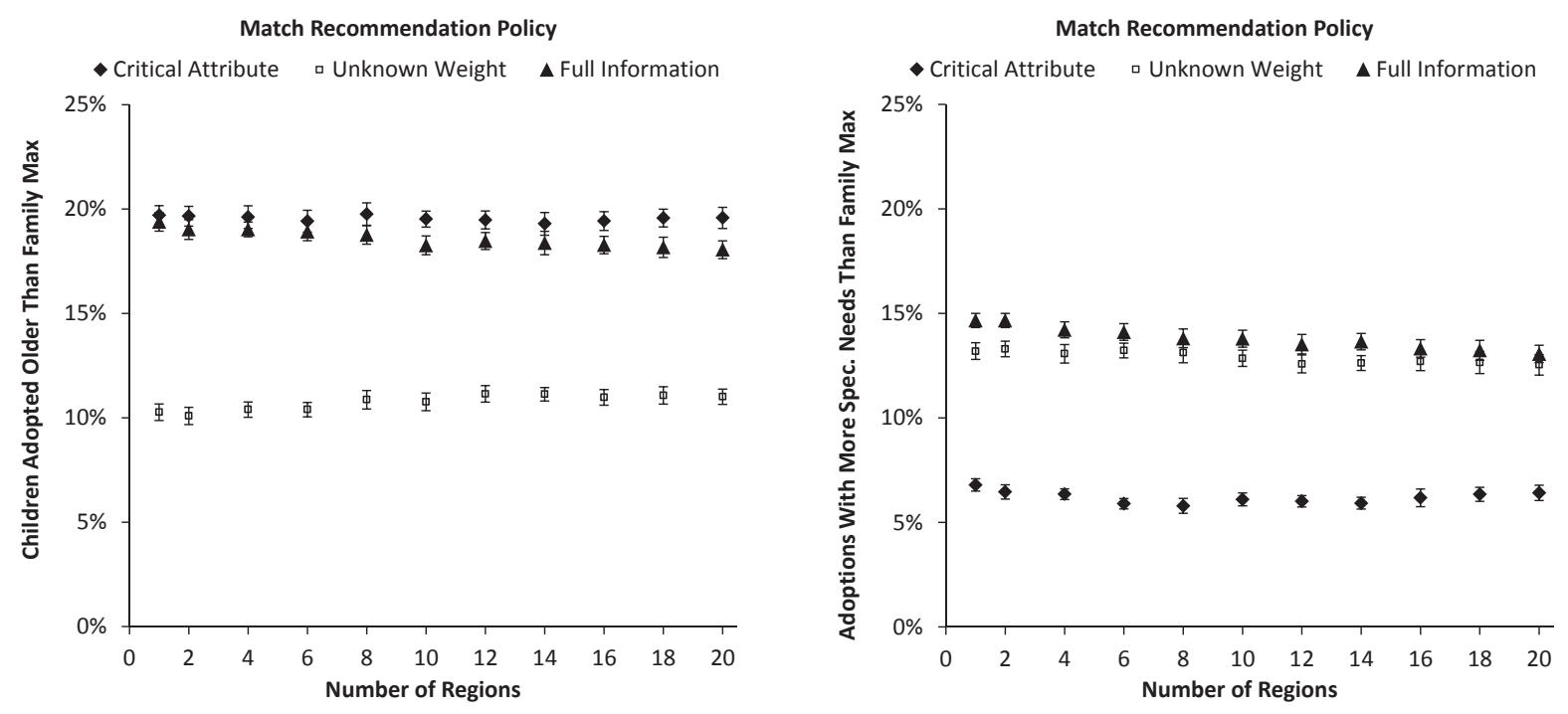

Figure A.4 Families' preferences are violated more frequently in adoptions for the Critical Attribute and Full Information policies than the Unknown Weight policy for a system with low attraction variability. Bands represent $95 \%$ confidence intervals.

The simulation was implemented in Java and relied upon the Java Simulation Library described in Rossetti (2008) for simulation functions.

\section{Results Related to Match Quality}

We additionally consider metrics that provide insights into the quality of matches for children and families. Specifically, we record the percentage of families who adopt a child with attribute values larger than the family's stated maximums (Figure A.4). We use these metrics to represent the quality of matches for children, especially in terms of the special needs attribute as a higher stated tolerance for special needs represents the increased ability of a family to accommodate a child's needs. We also record the average nominal family utility for adoptions, which we use to represent the quality of matches from the perspective of families (Figure A.5).

We first examine the UW policy, which assumes that families give equal weight to the age and special needs components of the utility function, and the case of low attraction variability. Under the UW policy, the percentage of families who adopt children with attributes above stated maximums is between $10.1 \%$ and $11.1 \%$ for the age attribute and between $12.5 \%$ and $13.3 \%$ for the special needs attribute, depending on the number of regions and attraction variability. Compared to the UW policy, the CA policy results in matches for which more families adopt children higher than their stated maximums more frequently for the 


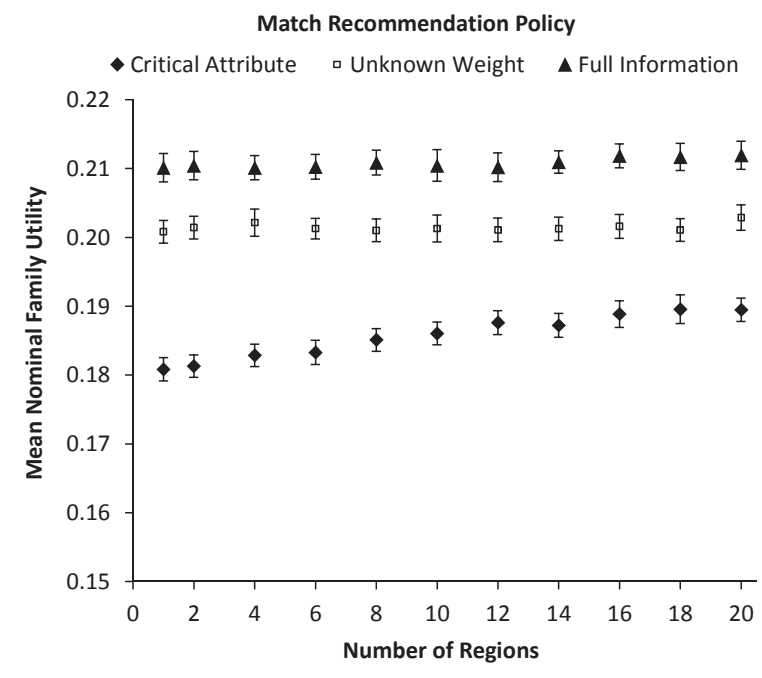

(a) Low Attraction Variability

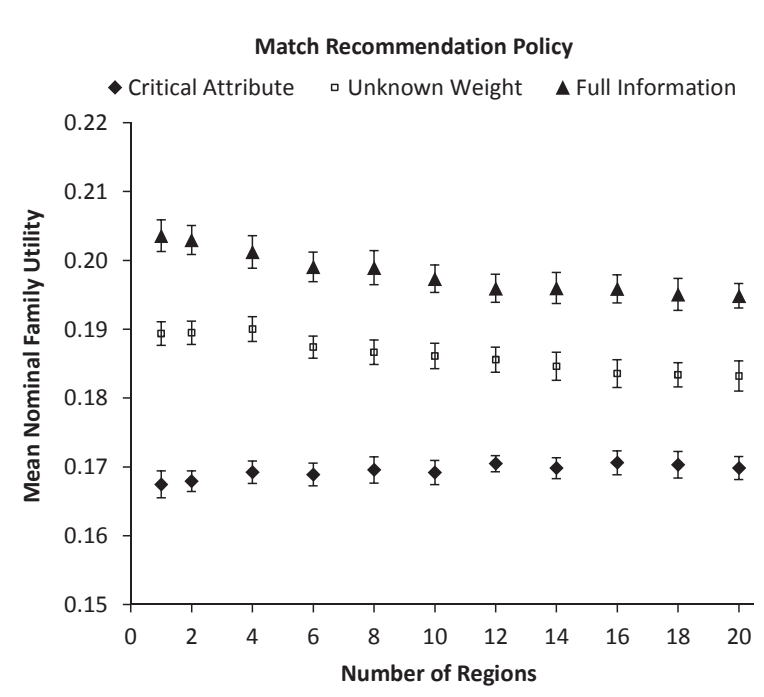

(b) High Attraction Variability

Figure A.5 The mean family nominal utility of successful matches increases with the quality of matching information. Bands represent $95 \%$ confidence intervals.

age attribute $(19.3 \%$ to $19.8 \%)$ and less frequently for the special needs attribute $(5.8 \%$ to $6.8 \%)$. Given that the penalty for exceeding a child's age in a utility function is relatively low for children who are younger than teenagers, the difference between the UW and CA policies can be explained by the practice of the CA policy to ignore the age attribute entirely if it is nominally less important than the special needs attribute.

For the FI policy, the percentage of families who adopt children with attribute values greater than their stated maximums is between $\mathbf{1 8 . 0 \%}$ and $19.4 \%$ for age, which is almost as high as the CA policy. For the special needs attribute, it is between $13.1 \%$ and $14.7 \%$, which is higher than the UW policy. This helps to explain the success of the FI policy with respect to the mean adoption rate; it uses knowledge of the weighting term $\alpha$ to violate families' preferences when relatively insignificant to propose plausible matches that have a greater chance of success. This explanation is supported by Figure A.5, which shows that the mean family utility increases from CA to UW and from UW to FI. We omit a corresponding chart for high attraction variability that shows a similar relationship between the three policies, although in all cases the percentage of families who adopt children with attribute values higher than their stated maximums is higher due to increased attraction variability.

The managerial insight regarding match quality is that improving the matching rate corresponds to more matches that violate families' stated age and special needs preferences when either of those preferences may be relatively less important to families. This highlights the importance of better understanding families' 
preferences as expressed through registration data and actual match rejection decisions. For example, allowing families to state a preference of "willing to accept training" for individual child special needs questions in registration data could allow PAE to better estimate how families weight questions. Similarly, PAE should expect to offer training more frequently to families on how to handle specific child special needs to maintain match quality for children.

\section{References}

Abdulkadiroğlu, A., T. Sönmez. 2003. School choice: A mechanism design approach. American Economic Review 93(3) 729-747.

Arikan, M., B. Ata, J. Friedewald, R. Parker. 2012. What drives the geographical differences in deceased donor organ procurement in the United States? Working paper, University of Kansas.

Ata, B., A. Skaro, S. Tayur. 2012. OrganJet: Overcoming geographical disparities in access to deceased donor kidneys in the United States. Working paper, Northwestern University.

Baccara, M., A. Collard-Wexler, L. Felli, L. Yariv. 2014. Child-adoption matching: Preferences for gender and race. American Economic Journal: Applied Economics 6(3) 133-158.

Biller, B., S. Ghosh. 2006. Multivariate input processes. B. L. Nelson, S. G. Henderson, eds., Handbooks in Operations Research and Management Science, vol. 13. Elsevier Science, Amsterdam, 123-153.

Children's Bureau, U.S. Department of Health and Human Services. 2014. Child welfare outcomes 2009-2012. http://www.acf.hhs.gov/programs/cb/resource/cwo-09-12. Online; accessed 16 April 2015.

Coles, P., J. Cawley, P. Levine, M. Niederle, A. Roth, J. Siegfried. 2010. The job market for new economists: A market design perspective. The Journal of Economic Perspectives 24(4) 187-206.

Gale, D., L. Shapley. 1962. College admissions and the stability of marriage. The American Mathematical Monthly 69(1) 9-15.

Hanna, M. D., R. G. McRoy. 2011. Innovative practice approaches to matching in adoption. Journal of Public Child Welfare 5(1) 45-66.

Howard, J., S. Brazin. 2011. Never too old: Achieving permanency and sustaining connections for older youth in foster care. Tech. rep., Evan B. Donaldson Adoption Institute, New York, NY. 
Kennan, J. 2006. A note on discrete approximations of continuous distributions. Unpublished manuscript.

Landes, E, R Posner. 1978. The economics of the baby shortage. Journal of Legal Studies 7 323-348.

Lee, S., M. Niederle, H. Kim, W. Kim. 2011. Propose with a rose? signaling in internet dating markets. Tech. rep., National Bureau of Economic Research.

PA Department of Human Services. 2015. Governor's 2015-2016 executive budget. http://www. dhs . state. pa.us/publications/budgetinformation/dhsbudget/index.htm. Online; accessed 16 April 2015.

Reilly, T. 2003. Transition from care: status and outcomes of youth who age out of foster care. Child Welfare: Journal of Policy, Practice, and Program 82(6) 727-746.

Rossetti, M. D. 2008. Java simulation library (JSL): an open-source object-oriented library for discrete-event simulation in Java. International Journal of Simulation and Process Modelling 4(1) 69-87.

Roth, A., E. Peranson. 1999. The redesign of the matching market for american physicians: Some engineering aspects of economic design. American Economic Review 89 748-780.

Roth, A., T. Sönmez, M. U. Ünver. 2005. Pairwise kidney exchange. Journal of Economic Theory 125 151-188. 